\title{
Potential pharmacologic treatments for COVID-19 patients: A review study
}

\author{
Zeinab Cheraghi ${ }^{1}$, Muhammadhosein Moradi $^{1}$, and Seyed Ali Ziai ${ }^{1}$ \\ ${ }^{1}$ Shahid Beheshti University of Medical Sciences
}

May 6, 2020

\begin{abstract}
A cluster of pneumonia cases of unknown origin was detected on December 31th which led to the discovery of coronavirus disease 2019 (COVID-19). Lungs are the primary site of involvement. SARS-COV-2, which is the causative agent, enters the alveolar cells using ACE2 as a receptor. Due to exposure of first cases to Wuhan's animal market, a zoonotic transmission was suspected. Further studies suggested human to human transmission through contact and droplets. Symptoms vary from asymptomatic to acute respiratory distress syndrome and death and cases are diagnosed based on clinical and laboratory findings. Currently, there is no definitive treatment or vaccine and different antiviral and other treatments are being tested as possible therapeutic agents with different mechanisms, for example, Chloroquine, hydroxychloroquine, azithromycin, favipiravir, umifenovir, ribavirin, Ivermectin, etc.
\end{abstract}

\section{Keywords}

SARS-CoV-2, COVID-19, ARDS, pharmacology, treatment, therapeutic options, chloroquine, hydroxychloroquine, azithromycin, favipravir, umifenovir, protease inhibitor, Mpro, remdesivir, monoclonal antibodies

\section{Introduction}

On December 31th, 2019, a group of pneumonia cases of unknown origin in the city of Wuhan in the Hubei Province in China was detected (1) which was later found out to be caused by a new emerging $\beta$-coronavirus, called 2019 novel coronavirus(2019-nCoV) or Severe acute respiratory syndrome coronavirus 2(SARS-CoV2) (2). On February $8^{\text {th }}, 2020$ pneumonia caused by this agent was afterward named Novel Coronavirus Pneumonia (NCP) (3) which was later changed to Coronavirus infection disease 2019(COVID-19). COVID19 starting in China, rapidly spread to other parts of the country and other nations (4) as well, with an exponential growth rate (5) so as until April $23^{\text {rd }}, 2020$ causing 2,544,792 confirmed cases and 175,694 deaths worldwide with the United States, Spain and Italy being affected the most (6). On January $30^{\text {th }}, 2020$ the world health organization declared a state of Public health emergency of international concern(PHEIC) for the sixth time (5), and on March 11 ${ }^{\text {th }}, 2020$ WHO assessed COVID-19 as a pandemic (7). SARS-CoV-2 is an enveloped, single-stranded, positive-sense, zoonotic member of $\beta$-coronaviruses $(4,8)$ which are known for causing more severe and lethal disease (4) as in previous major epidemics like severe acute respiratory syndrome(SARS) and middle-eastern respiratory syndrome(MERS) (2). The first cases had a history of being in touch with seafood or animal wholesale markets which raised the suspicion of zoonotic transmission $(9,10)$, and bats were assumed to be the reservoir host (11). The virus has an incubation period of 1 to 14 days, which for most cases, an incubation period of 4 days has been reported $(3,5,11)$ and can be transmitted through droplets, contaminated objects, and Human-to-Human contact whether being symptomatic or asymptomatic. COVID-19 confirmed cases are seen mostly among adults with children under 10 only accounting for $0.9 \%$ cases (12) and mostly being asymptomatic or having mild forms of the disease so having the capacity to act 
as carriers $(1,4)$. Patients mostly complain of fever, dry cough, and myalgia with associated laboratory and radiological findings. There is no specific treatment or vaccine for COVID-19 until now but several potential options under investigation. In this study we aim to have a look into potential pharmacologic treatment options for COVID-19.

\section{Method}

On March 11th, 2020 search engines such as Pubmed, Scopus, Clinicalkey, Cochrane library and Embase were used to search 'SARS2 and treatment', 'SARS2 and therapy', 'COVID 19 and therapy','covid19 and treatment' and 'COVID 19 and drug' resulting in a total of 68 articles which 29 articles were excluded due to repetition, irrelevancy, accessibility and language and 24 articles were selected based on a revision of abstracts from the 39 remaining articles. As follow up, another search was conducted on March 22nd 2020 on Pubmed search engine with the "COVID-19 and treatment" keyword, from which 43 articles were selected by titles among 347 results and 40 were chosen further by abstracts.

Finally, on April 5th 2020, the LitCovid engine was searched under the treatment tab from April 1st until April 5th and among 139 of a total of 614 results, 17 articles were selected by title which was further narrowed down to 14 due to repetition and irrelevancy based on abstracts. Kowsarpub was also searched for COVID19 and 14 articles were selected by title in the first step, among which 9 articles remained after further evaluation of abstracts. Pubmed was also searched for "COVID-19 AND favipiravir"," COVID-19 AND umifenovir", "COVID-19 AND azithromycin" and "COVID-19 AND plasma therapy" that resulted in 3,4,3 and 7 articles respectively. Later on, "covid-19 AND umifenovir" and "covid-19 AND azithromycin" were narrowed down to 3 and 2 results respectively due to repetition and a lack of compatibility. At last, new drug choices based on computational studies were obtained and articles' references were evaluated as well.

\section{Pathogenesis}

The main cells which are affected by COVID-19 seem to be alveolar type 2 cells which contain Angiotensin Converting Enzyme 2 (ACE 2) $(9,13)$. Being a member of $\beta$-coronaviruses, SARS-CoV-2 has the crown figure on electron microscopic investigation. The spike(S) proteins, the spikes of the crown, bind to ACE2 and facilitates viral attachment through cellular surface into target cells (2). From proteins among members of this family, ACE and ACE2 are of value. Angiotensin II, binds to AT1R on alveolar cells and activates it. ACE converts angiotensin I (produced by enzymatic actions of renin on angiotensinogen) into angiotensin II, a vasoconstrictor agent. ACE2 on the other hand opposes ACE's function by inactivating angiotensin II and converting it to angiotensin 1-7, a heptapeptide with vasodilator functions. SARS-CoV-2 uses ACE2, as the receptor binding domain (13). Alveolar damage caused by the virus leads to an increase in proinflammatory cytokines (14)causing an interstitial inflammatory infiltration dominated by lymphocytes which contributes to more infiltration ultimately resulting in pneumocytes desquamation and hyaline membrane formation indicating acute respiratory distress syndrome (ARDS) (15).

\section{Transmission}

The first detected cases of coronavirus had a history of contact with bats which caused the suspicion of zoonotic measures (11). Further studies suggested the presence of viruses in body fluids thus droplets and body fluids including sputum and blood could be a means of transmission so precautions should be taken towards blood donation $(1,5)$. Direct human-to-human transmission and airborne transmission in small closed-areas have also been suspected (10). There is no evidence suggesting vertical mother to child transmission (16).

\section{Clinical manifestation}

COVID-19 is an acute respiratory disease. It is present in mild, moderate, and severe forms which, affects all groups of people but it is mostly seen in adults (10). Children usually are asymptomatic or manifest mild gastrointestinal symptoms $(3,12)$. Adults are symptomatic featuring 3 common symptoms of fever, dry cough, and myalgia. Other symptoms including chills, shortness of breath, chest pain, malaise, fatigue, headache, nasal congestion, rhinorrhea $(3,10,17)$ and GI symptoms such as nausea, abdominal pain and 
diarrhea (18) and dyspnea which is a sign of disease progression (4), has been reported within less than a week. During the second and third weeks of symptomatic infection, signs of pneumonia have been discovered within $75 \%$ of patients. On physical examination patients based on the severity of the disease have various findings, ranging from normal findings to respiratory distress, tachycardia, and fever. There has been no specific diagnostic laboratory findings but among the tests, elevated levels of Aspartate aminotransferase (AST), Alanine aminotransferase (ALT), lactate dehydrogenase (LDH), C-reactive protein (CRP), and lactate (19, 20) have been found alongside a leukopenia consisting of neutropenia and lymphopenia which is a criteria of poor prognosis $(10,15)$. Imaging studies with computed tomography $(\mathrm{CT})$ has been used as a complementary diagnostic tool as well and findings are usually seen, 10 days after onset of the symptoms (21). These studies revealed a range of findings from no findings in a few cases to unilateral and bilateral pulmonary engagement (22). Increased bilateral para hilar infiltration and ill-defined irregular patchy consolidations with increased thickening of reticular or interlobular septa were seen (3,23). Bilateral peripheral Grand glass opacities (24) may also be seen in CT images (25). In some cases some uncommon findings such as small bilateral pleural effusions and mediastinal lymphadenopathy was also detected (21).

\section{Diagnosis}

Clinical manifestation such as cough, fever and myalgia are being used as a primitive screening tool determining the necessity of referral to medical centers. A definitive diagnosis is established based on laboratory reports. Sputum, nasopharyngeal swabs, oropharyngeal swabs, blood, urine and feces $(10,17)$ were used as samples for diagnosis and treatment procedure follow up. Currently there is no definitive serologic diagnostic laboratory finding. Diagnosis is established based on the presence of viral genome. Real-time reverse transcription polymerase chain reaction (rRT-PCR) is mostly used for this cause along with RNA-based metagenomic sequencing and CRISPR-based technique $(1,5,26)$. Besides IgM/IgG anti-SARS-CoV-2 antibodies can also be used as a means of diagnosis (27). Imaging studies are also a promising diagnostic tool.

\section{Pharmacologic management}

Various treatments are assorted based on different stages of coronavirus entrance, virus replication, body reactions to the virus, and the effects of the Immune system on the virus.

\section{1- Inhibition of the coronavirus entrance into the body and binding to the receptor}

SARS-CoV-2 binds to the ACE2 and dysregulates the renin-angiotensin system (13). SARS-CoV-2 attaches to its receptor using its surface spike (S) protein (8). Fusion and entrance are based on the binding of the $\mathrm{S}$ protein to the ACE2 receptor on the cells. Due to the ACE2 role as a viral receptor, there are some suggestions to use AT1R antagonists (ARB) in COVID-19 treatment (5). Experiments have proved that binding the coronavirus to its receptors on the cardiac cells, decreased ACE2 enzyme expression. This action results in ACE enzyme activation and produces angiotensin. Considering these findings, taking AT1R antagonists seems to be a suitable option. Although AT1R antagonists have rarely shown adverse drug reactions But hypotension is reporting in some of the SARS-CoV patients (13). Both angiotensin converting enzyme inhibitors (ACEI) and angiotensin II receptor blockers (ARBs) drugs have the same therapeutic effects and adverse effects; for example: Severe hypotension after initial doses, acute renal failure $(28,29)$. Chloroquine was mentioned to interfere with coronavirus-ACE2 connection. Recombinant human monoclonal antibodies $(\mathrm{mAb})$ could be used to interfere with virus binding according to Zhang et al. The mAb could efficiently neutralize SARS-CoV and inhibit syncytia formation between cells expressing the $\mathrm{S}$ protein and those expressing the SARS-CoV receptor ACE2 (8). Peptide-based vaccines against coronavirus can not be utilized in subsequent epidemics considering mutations in the virus genome (13).

\section{2- Inhibition of the coronavirus uncoating}

Chloroquine has shown in-vitro effects such as inhibition of uncoating and glycosylation in various viruses, so it is an appropriate case study for COVID-19 treatment according to accomplished clinical trials conducted in China (5). In chloroquine administration, caution must be taken since profound side effects such as 
retinopathy may proceed. Chloroquine co-administration with lopinavir/ritonavir causes prolonged QT interval, torsades de pointes, and may result in sudden death (30). Pruritus is common. Rare adverse effects include hemolysis in glucose- 6-phosphate dehydrogenase (G6PD)-deficient persons, agranulocytosis, hypotension, and electrocardiographic changes(28).

\section{3- Inhibition of viral replication}

Hydroxychloroquine inhibits virus replication in different steps: inhibition of the fusion of the virus to its receptor, inhibition of the nucleic acid replication, virus assembly, and virus release (32). In a study, the therapeutic plan was using hydroxychloroquine for 5 days; the results supported the application for SARSCoV-2 infection treatment (31). Another study which used hydroxychloroquine for 3 to 6 days, showed that it could decrease viral load reduction in COVID-19 patients. Also, a combination of hydroxychloroquine and azithromycin had synergic effects on viral elimination $(32,33)$. Other antiviral treatments are as follows:

a) Ribavirin and interferon-, are virus replication inhibitors in animals and human cell lines. Adverse effects of ribavirin include hemolytic anemia and decreased hemoglobin. It is contraindicated in pregnant patients.

b) Lopinavir/ritonavir (Kaletra) : Lopinavir/ritonavir (LPV/RTV) is a combination of lopinavir and ritonavir (34). RTV inhibits the CYP3A-mediated metabolism of LPV, thereby increasing the serum concentration of LPV(35). This combination reduces coronavirus viral loads remarkably(36). LPV inhibits polyprotein processing in $\mathrm{CoV}(34)$. SARS-CoV2 and SARS-CoV share a $79.5 \%$ sequence identity. LPV is useful for treating SARS and MERS patients, so it may be useful in COVID-19 patients, too. Chu et al have found out that the use of LPV/ RTV with ribavirin showed better results in the treatment of SARS (34). Deng et al found that 16 patients who received arbidol and LPV/RTV had better clinical manifestation than LPV/RTV only (35). Caution should be taken when taking Kaletra, since it can result in upper respiratory tract infection, vasodilation, thrombocytopenia, neutropenia, stevens-Johnson syndrome, transaminase increase, and a possible increase in myocardial infarction $(28,37)$.

c) Remdesivir : Remdesivir is an adenosine triphosphate analogue and a broad-spectrum antiviral which affects viral RNA polymerase and terminates RNA transcription. (38-40). It was primarily developed for Ebola treatment and demonstrated effectiveness against SARS and MERS in animal models. A combination of remdesivir (RDV) and interferon- $\beta$ have shown stronger antiviral activity against MERS-CoV to that of Lopinavir/ritonavir and IFN- $\beta$. Also, remdesivir unlike LPV/RTV-IFN- $\beta$, could reduce lung viral loads against MERS in animal study $(8,41)$. It is currently under several clinical trials for assessing its efficacy against COVID-19. Toxicity and adverse effects are under investigation.

d) Oseltamivir: The strategy that has been tried so far showed that 75 patients were administrated $75 \mathrm{mg}$ oseltamivir, $500 \mathrm{mg}$ ritonavir, $500 \mathrm{mg}$ lopinavir, and the intravenous administration of $0.25 \mathrm{~g}$ ganciclovir for 3-14 days twice a day (11). In a case series in Wuhan, China, most of the patients have been treated by oseltamivir, moxifloxacin, ceftriaxone, and azithromycin. Some of them received glucocorticoid therapy. Discharge rate of $34.1 \%$ and the mortality rate of $4.3 \%$ have been observed (17). The benefits of Oseltamivir on the course of treatment was unknown (42).

e) Favipiravir is a broad-spectrum antiviral drug that inhibits viral replication. Favipiravir treatment increased the survival rate and decreased viral load. Favipiravir has potential drug-drug interactions and in patients with basic diseases have to pay attention(43). Favipiravir is contraindicated in pregnancy(44).

\section{f) Protease inhibitors}

Cinanserin, a known serotonin antagonist, which also showed hopeful SARS-CoV inhibitory features, is another possible option to be considered. It could inhibit the 3 chymotrypsin-like protease and could inhibit SARS-CoV replication (45). The 3C-L protease is encoded in SARS-CoV2. Therefore, Cinanserin may be an effective therapy for the COVID-19 infection. Also, flavonoids like: Herbacetin, rhoifolin, and pectolinarin are $3 \mathrm{C}$-like protease inhibitors (8).

Nelfinavir is an HIV-1 protease inhibitor previously used in HIV treatment that acts through inhibition of 
3C-L protein (45).

Carfilzomib is an approved anti-cancer drug which by inhibition of proteasome shows potential for inhibiting COVID-19 protease (46).

Indinavir: It is an anti-HIV drug that based on molecular studies can also be used against SARS-COV-2 by potentially inhibiting the viral protease. Adverse effects are mostly of gastrointestinal and hepatic symptoms $(47,48)$.

Danoprevir: It is another HCV NS3/4 protease inhibitor has also shown potential according to the studies. It is also being used for Hepatitis $\mathrm{C}$ treatment. Studies reveal a pharmacokinetic boost in co-administration of danoprevir with ritonavir (49).

Ledipasvir and velpatasvir, NS5A protein inhibitors of HCV have been proposed for further investigation due to virtual screening studies. Ledipasvir and velpatasvir in combination withsofosbuvir have been used as a potent anti-hepatitis $\mathrm{C}$ treatment. They may also cause headache, fatigue, and nausea as adverse effects (50).

SARS-CoV-2 M protein is the main enzyme utilized by the virus for replication, thus making it an appealing target. Studies suggest multiple agents that may or may not be approved by the FDA. For example:Telaprevir and boceprevi $\mathrm{r}$. These are $\alpha$-ketoamide drugs that target the main protease of coronaviruses and $3 \mathrm{C}$ protease of enteroviruses and have a crucial role in the treatment of hepatitis C (51).

\section{g) Immunoenhancers}

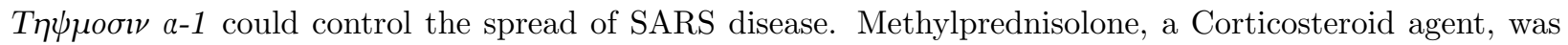
often used during the acute respiratory distress syndrome(ARDS) and it Destroyed thymocytes. So, it is better to use thymosin $\alpha 1$ at the early illness, before ARDS happens(52).

Levamisole: This drug has different effects on the immune system based on the dosing and the time of administration; immunostimulant agent or immunosuppressive agent. A combination of levamisole and ascorbic acid was reported to be a lymphocyte enhancer (53).

h) Vitamins and supplements

$B$ vitamins : These Vitamins have a role in the immune system and B vitamin deficiency could result in a weak immune system. So resolving B vitamin deficiencies is important for strengthening the body against the coronavirus(8).

Vitamin $C$ : Vitamin $\mathrm{C}$ is necessary for immune system functions (54). Lower respiratory tract infection could be a result of COVID-19 disease, so vitamin $\mathrm{C}$ could be an effective option for the treatment of this infection (8).

Vitamin D3 : Patients with a weak immune system as a result of vitamin D3 deficiency, are at a higher risk to infect to COVID-19 (55).

omega-3 Polyunsaturated fatty acids(PUFA)-derived lipid mediator : This lipid mediator inhibited influenza virus replication strongly (8).

Selenium: Selenium deficiency affects the immune system response and the virus elimination (56).

Ebselen: It' an anti-oxidant agent with anti-inflammatory and cytoprotective properties $(45,57)$.

Glycyrrhizin, a component of licorice roots, is a traditional Chinese herbal medicine. Glycyrrhizin has inhibitory effects on SARS replication.

Baicalin, another Chinese herb was also found to be a SARS-CoV inhibiting and immune system enhancer.

Shikonin is also another herbal medicine showing promising results (45). 
Estradiol and phytoestrogen : Epidemiologic studies reveal men undergo a higher rate of morbidity and mortality in comparison to females after SARS-CoV infection. However an increase was reported in the number of female mice mortality after ovariectomy or administration of estrogen receptor antagonist (8).

\section{i) Miscellaneous agents}

Bictegravir an integrase inhibitor is a recently approved drug for HIV which can cause gastrointestinal, headache, and hepatic side effects with dofetilide and rifampin contraindication $(45,58,59)$.

Arbidol (Umifenovir): Arbidol prevents virus entrance into the cells through inhibition of the interaction between virus and plasma membrane (60). Arbidol also can induce interferons and activate macrophages and be an immune enhancer (61). Some patients received arbidol treatment at a dose of $0.4 \mathrm{~g}$ three times a day. Arbidol treatment improved the discharging rate and decreased the mortality rate (62).

Prulifloxacin is a fluoroquinolone with CYP1A2 inhibitory property which also showed anti-SARS-CoV-2 potential based on computational studies $(63,64)$.

Disulfiram an aldehyde dehydrogenase inhibitory agent, used in the treatment of alcoholism shows promise in inhibiting SARS-COV-2. Disulfiram has little side effects but causes drug-drug interactions with multiple drugs including phenytoin, isoniazid, oral anticoagulants. Alcohol consumption in a patient of disulfiram medication can lead to vomiting, sweating, throbbing headache, hypotension, and confusion. Its safety is not established in pregnancy $(45,65)$.

Carmofur is a known anti-neoplastic agent used in colorectal and breast cancers. It is known to cause leukoencephalopathy (45).

Tideglusib a glycogen synthase kinase 3 inhibitor with anti-inflammatory features that was primarily developed for Alzheimer's disease $(45,66)$.

1-methylpropyl 2-imidazolyl disulfide (PX-12) is an inhibitor of thioredoxin-1 (Trx-1) which is associated with colorectal, gastric, and lung cancer $(45,67)$.

$T D Z D-8$ is a thiadiazolidine derivative that has potential inhibitory effects on viral M protein. $(45,68)$

Ivermectin is an anti-protozoal drug that has been in use since 1970, is another option to be considered. According to studies, it has shown promising potential in laboratory studies and also had exhibited potential infection control in similar infections such as West Nile virus (69).

Moexipril, a long-acting ACE inhibitor prodrug;Daunorubicin and Mitoxantrone, anthracycline antibiotics which are most widely used chemotherapy drugs(65); Bepotastine, a non-sedating histamine 1 receptor antagonist(70); rosuvastatin, an $\mathrm{HMG-CoA}$ reductase inhibitor which in some cases can cause hepatic toxicity and Atovaquone, (65) the antimalarial drug that is also used in pneumonia $P$. jiroveci $(71)$ are also therapeutic options to be nominated for further investigations based on recent studies.

\section{4- Treatment of complications mediated by virus replication}

As the cytokine storm develops through the body, it causes shock and hypoxemia. Also, Antimicrobial and antifungal agents could be administered for elongated illness course. Application of prebiotics and Resolving nutritional deficiencies could be used alongside the main therapeutics. ARDS and difficulty in breathing are COVID-19 symptoms in which nitric oxide could help in regulating the function of the airways and inhibiting the synthesis of viral protein and RNA (8). An effective way is to administer a combination, including anti-shock, antivirus, anti-secondary infection, anti-hypoxemia, and maintenance of water, electrolyte and acid-base balance, and micro ecological balance and immune enhancement. It could effectively reduce the mortality rate in COVID-19 patients. (72).

Azithromycin, a macrolide and an erythromycin derivative, with unique pharmacologic properties and prolonged half-life, has been a part of the treatment options for community-acquired pneumonia. It can be used alone or in combination with other antimicrobial agents, depending on the history and severity 
of the disease(29). However, like other macrolides, through affecting potassium ion channels, prolongation of QT interval may happen and recent studies revealed an association of increased risk of cardiac death (28). Taking azithromycin may cause increased lactate dehydrogenase, thrombocythemia, lymphocytopenia (37). In a retrospective study of 85 fatal cases, most patients received antibiotics (Meropenem), antiviral (Arbidol), and glucocorticoid treatments. The administration of multiple antibiotics did not decrease the mortality rate. This article did not mention azithromycin(73).

\section{5- Managing Cytokine Release Syndrome Associated to coronavirus}

Regarding the importance of immune system functions against coronavirus, the WHO and CDC don't recommend using corticosteroids routinely in early COVID-19 illness treatment plan unless there are other indications for their usage(42). Whilst, the use of immunosuppressants (e.g. tocilizumab) is not ideal either, as it can suppress the immune system and lead to an increased risk of infection (31). In severely ill patients the treatment of cytokine storm has become an important part of therapy. Tocilizumab is a blocker of the IL-6 receptor, which is a key molecule in cytokine release syndrome (74). Taking tocilizumab may cause hypertension, peripheral edema, leukopenia, thrombocytopenia, and upper respiratory tract infection in some patients (37).

Convalescent Plasma Therapy is another way of managing the cytokine storm. Therapeutic Plasma Exchange (TPE) is not a novel approach as in 2014 it has been used against the Ebola virus. TPE has been kept as the last resort and previous studies confirmed a reduced mortality rate and hospital stay in patients receiving this treatment during SARS and MERS epidemics $(75,76)$. Convalescent plasma acquired from patients with severe disease might have higher titers of antibodies due to more intense immune system response and so can last longer periods (76). This medication seems to act through the suppression of viremia and managing the cytokine storm $(27,77)$. TPE also seems to reset the hypercoagulable state and stabilize the endothelial membranes (77). Since viremia peaks in the first week of most viral illnesses, it has been suggested that early administration of TPE, can fill the gap between the entrance of the virus and primary immune response and can be more effective in the first weak rather than later (75). No serious adverse drug reaction has been reported $(75,76)$. However, as TPE is co-administrated with other therapeutic regimens, the antibody levels and the efficacy of this method may be confounded by factors such as supportive care, antiviral agents, steroids, and intravenous immunoglobulin and remains unclear (76).

\section{Conclusion}

Currently, there is no FDA approved pharmacologic therapy. Disease management is based on prevention and quarantine. Pharmacologic therapy choices are all off-label. Several antiviral components are also being administered as off-label therapy. Oseltamivir, Hydroxychloroquine, and Kaletra are currently being used. Monoclonal Antibodies can also be a treatment choice but are restricted by viral mutations and peptide base changes. Vitamins B, C, and D appear to be useful in enhancing the immunity system of patients with deficiencies. Zinc and Selenium can also be used for that purpose. Side effects and contraindications must also be taken into account since higher mortality rates are reported in patients with underlying disorders. The use of corticosteroids must be limited to specific cases otherwise immunity system's suppression could lead to exacerbation of the disease. Depending on SARS-CoV and SARS-CoV-2 high sequence homology, relevant data can be utilized in choosing therapy. Antivirals seem to be the choice of therapy; along with monoclonal antibodies. Clinical Trials and more studies need to be conducted to determine efficacy and appropriate dosage and therapy period.

Conflict of interest

The authors have no conflicts of interest relevant to this article.

Funding

This research did not receive any specific grant from funding agencies in the public, commercial, or not-forprofit sectors. 


\section{References}

1. Chang L, Yan Y, Wang L. Coronavirus Disease 2019: Coronaviruses and Blood Safety. Transfusion Medicine Reviews. 2020.

2. Hoffmann M, Kleine-Weber H, Schroeder S, Kruger N, Herrler T, Erichsen S, et al. SARS-CoV-2 Cell Entry Depends on ACE2 and TMPRSS2 and Is Blocked by a Clinically Proven Protease Inhibitor. Cell. 2020 .

3. Xu YH, Dong JH, An WM, Lv XY, Yin XP, Zhang JZ, et al. Clinical and computed tomographic imaging features of novel coronavirus pneumonia caused by SARS-CoV-2. The Journal of infection. 2020.

4. Velavan TP, Meyer CG. The COVID-19 epidemic. Tropical Medicine and International Health. 2020;25(3):278-80.

5. Lai CC, Shih TP, Ko WC, Tang HJ, Hsueh PR. Severe acute respiratory syndrome coronavirus 2 (SARSCoV-2) and coronavirus disease-2019 (COVID-19): The epidemic and the challenges. International Journal of Antimicrobial Agents. 2020.

6. Organization WH. Corona virus statistics. 2020.

7. Organization WH. Pandemic state.

8. Zhang L, Liu Y. Potential Interventions for Novel Coronavirus in China: A Systemic Review. Journal of medical virology. 2020.

9. Lin L, Jiang X, Zhang Z, Huang S, Zhang Z, Fang Z, et al. Gastrointestinal symptoms of 95 cases with SARS-CoV-2 infection. Gut. 2020.

10. Xu XW, Wu XX, Jiang XG, Xu KJ, Ying LJ, Ma CL, et al. Clinical findings in a group of patients infected with the 2019 novel coronavirus (SARS-Cov-2) outside of Wuhan, China: retrospective case series. BMJ (Clinical research ed). 2020;368:m606.

11. Rothan HA, Byrareddy SN. The epidemiology and pathogenesis of coronavirus disease (COVID-19) outbreak. Journal of Autoimmunity. 2020.

12. Cao Q, Chen Y-C, Chen C-L, Chiu C-H. SARS-CoV-2 infection in children: Transmission dynamics and clinical characteristics. Journal of the Formosan Medical Association= Taiwan yi zhi. 2020.

13. Gurwitz D. Angiotensin receptor blockers as tentative SARS-CoV-2 therapeutics. Drug development research. 2020.

14. Fu Y, Cheng Y, Wu Y. Understanding SARS-CoV-2-Mediated Inflammatory Responses: From Mechanisms to Potential Therapeutic Tools. Virologica Sinica. 2020.

15. Xu Z, Shi L, Wang Y, Zhang J, Huang L, Zhang C, et al. Pathological findings of COVID-19 associated with acute respiratory distress syndrome. The Lancet Respiratory Medicine. 2020.

16. Qiao J. What are the risks of COVID-19 infection in pregnant women? The Lancet. 2020.

17. Young BE, Ong SWX, Kalimuddin S, Low JG, Tan SY, Loh J, et al. Epidemiologic Features and Clinical Course of Patients Infected With SARS-CoV-2 in Singapore. Jama. 2020.

18. Song Y, Liu P, Shi XL, Chu YL, Zhang J, Xia J, et al. SARS-CoV-2 induced diarrhoea as onset symptom in patient with COVID-19. Gut. 2020.

19. Xu J, Zhao S, Teng T, Abdalla AE, Zhu W, Xie L, et al. Systematic Comparison of Two Animal-toHuman Transmitted Human Coronaviruses: SARS-CoV-2 and SARS-CoV. Viruses. 2020;12(2). 
20. Huang WH, Teng LC, Yeh TK, Chen YJ, Lo WJ, Wu MJ, et al. 2019 novel coronavirus disease (COVID-19) in Taiwan: Reports of two cases from Wuhan, China. Journal of Microbiology, Immunology and Infection. 2020.

21. Gross A, Thiemig D, Koch FW, Schwarz M, Glaser S, Albrecht T. CT appearance of severe, laboratoryproven coronavirus disease 2019 (COVID-19) in a Caucasian patient in Berlin, Germany. RoFo : Fortschritte auf dem Gebiete der Rontgenstrahlen und der Nuklearmedizin. 2020.

22. Sun D, Li H, Lu XX, Xiao H, Ren J, Zhang FR, et al. Clinical features of severe pediatric patients with coronavirus disease 2019 in Wuhan: a single center's observational study. World journal of pediatrics : WJP. 2020.

23. Wang D, Hu B, Hu C, Zhu F, Liu X, Zhang J, et al. Clinical Characteristics of 138 Hospitalized Patients With 2019 Novel Coronavirus-Infected Pneumonia in Wuhan, China. Jama. 2020.

24. Liu KC, Xu P, Lv WF, Qiu XH, Yao JL, Gu JF, et al. CT manifestations of coronavirus disease-2019: A retrospective analysis of 73 cases by disease severity. European journal of radiology. 2020;126:108941.

25. Ding Q, Lu P, Fan Y, Xia Y, Liu M. The clinical characteristics of pneumonia patients co-infected with 2019 novel coronavirus and influenza virus in Wuhan, China. Journal of medical virology. 2020.

26. Cheng SC, Chang YC, Fan Chiang YL, Chien YC, Cheng M, Yang CH, et al. First case of Coronavirus Disease 2019 (COVID-19) pneumonia in Taiwan. Journal of the Formosan Medical Association. 2020.

27. Ma J, Xia P, Zhou Y, Liu Z, Zhou X, Wang J, et al. Potential effect of blood purification therapy in reducing cytokine storm as a late complication of severe COVID-19. Clinical immunology (Orlando, Fla). 2020:108408.

28. Katzung BG. Basic\&Clinical pharmacology fourteenth Edition. 2018:833.

29. Jameson F, Kasper,Hauser,Longo,Loscalzo. Harrison's Principle of Internal Medicine 20th Edition. 2018:913.

30. Sahraei Z, Shabani M, Shokouhi S, Saffaei A. Aminoquinolines against coronavirus disease 2019 (COVID19): chloroquine or hydroxychloroquine. International Journal of Antimicrobial Agents. 2020:105945.

31. Yao X, Ye F, Zhang M, Cui C, Huang B, Niu P, et al. In Vitro Antiviral Activity and Projection of Optimized Dosing Design of Hydroxychloroquine for the Treatment of Severe Acute Respiratory Syndrome Coronavirus 2 (SARS-CoV-2). Clinical infectious diseases : an official publication of the Infectious Diseases Society of America. 2020.

32. Gao J, Tian Z, Yang X. Breakthrough: Chloroquine phosphate has shown apparent efficacy in treatment of COVID-19 associated pneumonia in clinical studies. Bioscience trends. 2020.

33. Gautret P, Lagier JC, Parola P, Hoang VT, Meddeb L, Mailhe M, et al. Hydroxychloroquine and azithromycin as a treatment of COVID-19: results of an open-label non-randomized clinical trial. Int J Antimicrob Agents. 2020:105949.

34. Yao TT, Qian JD, Zhu WY, Wang Y, Wang GQ. A Systematic Review of Lopinavir Therapy for SARS Coronavirus and MERS Coronavirus-A Possible Reference for Coronavirus Disease-19 Treatment Option. Journal of medical virology. 2020.

35. Deng L, Li C, Zeng Q, Liu X, Li X, Zhang H, et al. Arbidol combined with LPV/r versus LPV/r alone against Corona Virus Disease 2019: A retrospective cohort study. The Journal of infection. 2020.

36. Lim J, Jeon S, Shin HY, Kim MJ, Seong YM, Lee WJ, et al. Case of the index patient who caused tertiary transmission of coronavirus disease 2019 in Korea: The application of lopinavir/ritonavir for the treatment of COVID-19 pneumonia monitored by quantitative RT-PCR. Journal of Korean Medical Science. $2020 ; 35(6)$. 
37. Post TW. Kaletra: UptoDate in Waltham,MA; 2020, 7 April.

38. Database D. Remdesivir 2020,April 14 [Available from: https://www.drugbank.ca/drugs/DB14761.

39. Kruse RL. Therapeutic strategies in an outbreak scenario to treat the novel coronavirus originating in Wuhan, China. F1000Research. 2020;9:72.

40. Dong L, Hu S, Gao J. Discovering drugs to treat coronavirus disease 2019 (COVID-19). Drug discoveries \& therapeutics. 2020;14(1):58-60.

41. Lu H. Drug treatment options for the 2019-new coronavirus (2019-nCoV). Biosci Trends. 2020;14(1):6971.

42. Columbus C, Brust KB, Arroliga AC. 2019 novel coronavirus: an emerging global threat. Baylor University Medical Center Proceedings. 2020.

43. Du YX, Chen XP. Favipiravir: pharmacokinetics and concerns about clinical trials for 2019-nCoV infection. Clinical pharmacology and therapeutics. 2020.

44. DrugBank Database. Favipiravir 2020, 7 April [Available from: https://www.drugbank.ca/drugs/DB12466.

45. Jin Z, Du X, Xu Y, Deng Y, Liu M, Zhao Y, et al. Structure of Mpro from COVID-19 virus and discovery of its inhibitors. bioRxiv. 2020.

46. Junmei W. Fast Identification of Possible Drug Treatment of Coronavirus Disease -19 (COVID-19) Through Computational Drug Repurposing Study2020.

47. Ted W. Post. Indinavir2020,April 11.

48. Chang Y-C, Tung Y-A, Lee K-H, Chen T-F, Hsiao Y-C, Chang H-C, et al. Potential therapeutic agents for COVID-19 based on the analysis of protease and RNA polymerase docking. 2020.

49. Chen H, Zhang Z, Wang L, Huang Z, Gong F, Li X, et al. First Clinical Study Using HCV Protease Inhibitor Danoprevir to Treat Naive and Experienced COVID-19 Patients. medRxiv. 2020:2020.03.22.20034041.

50. Chen YW, Yiu C-PB, Wong K-Y. Prediction of the SARS-CoV-2 (2019-nCoV) 3C-like protease (3CL (pro)) structure: virtual screening reveals velpatasvir, ledipasvir, and other drug repurposing candidates. F1000Research. 2020;9:129-.

51. Zhang L, Lin D, Kusov Y, Nian Y, Ma Q, Wang J, et al. $\alpha$-Ketoamides as Broad-Spectrum Inhibitors of Coronavirus and Enterovirus Replication: Structure-Based Design, Synthesis, and Activity Assessment. Journal of medicinal chemistry. 2020:acs.jmedchem.9b01828.

52. Baumann CA, Badamchian M, Goldstein AL. Thymosin $\alpha 1$ antagonizes dexamethasone and CD3-induced apoptosis of $\mathrm{CD} 4+\mathrm{CD} 8+$ thymocytes through the activation of cAMP and protein kinase $\mathrm{C}$ dependent second messenger pathways1This work is supported, in part, by a grant from SciClone Pharmaceuticals, San Mateo, California, to M. Badamchian and a pre-doctoral fellowship from the Philip Morris Companies Inc. to C.A. Baumann.1. Mechanisms of Ageing and Development. 1997;94(1):85-101.

53. M I Joffe NRS, and A R Rabson. Lymphocyte subsets in measles. Depressed helper/inducer subpopulation reversed by in vitro treatment with levamisole and ascorbic acid. J Clin Invest. 1983;72(3):971-80.

54. Hemilä H. Vitamin C and SARS coronavirus. The Journal of antimicrobial chemotherapy. 2004;52:104950.

55. Nonnecke BJ, McGill JL, Ridpath JF, Sacco RE, Lippolis JD, Reinhardt TA. Acute phase response elicited by experimental bovine diarrhea virus (BVDV) infection is associated with decreased vitamin D and E status of vitamin-replete preruminant calves1. Journal of Dairy Science. 2014;97(9):5566-79. 
56. Guillin OM, Vindry C, Ohlmann T, Chavatte L. Selenium, Selenoproteins and Viral Infection. Nutrients. 2019;11(9):2101.

57. Database D. Ebselen 2020,March 1 [Available from: https://www.drugbank.ca/drugs/DB12610.

58. Post TW. Bictegravir: UpToDate; 2020, April 12.

59. Database D. Bictegravir 2020,March 1 [Available from: https://www.drugbank.ca/drugs/DB11799.

60. Villalain J. Membranotropic effects of arbidol, a broad anti-viral molecule, on phospholipid model membranes. The journal of physical chemistry B. 2010;114(25):8544-54.

61. Liu Q, Xiong H-r, Lu L, Liu Y-y, Luo F, Hou W, et al. Antiviral and anti-inflammatory activity of arbidol hydrochloride in influenza A (H1N1) virus infection. Acta Pharmacologica Sinica. 2013;34(8):1075-83.

62. Wang Z, Yang B, Li Q, Wen L, Zhang R. Clinical Features of 69 Cases with Coronavirus Disease 2019 in Wuhan, China. Clinical infectious diseases : an official publication of the Infectious Diseases Society of America. 2020.

63. Alex Z, Vladimir A, Alexander Z, Bogdan Z, Victor T, Dmitry S. B, et al. Potential COVID-2019 3C-like Protease Inhibitors Designed Using Generative Deep Learning Approaches2020.

64. Database D. Prulifloxacin 2020,March 1 [Available from: https://www.drugbank.ca/drugs/DB11892.

65. Katzung BG. Basic\&Clinical pharmacology fourteenth Edition2018 April 9th 2020. 833 p.

66. Database D. Tideglusib 2020,March 1 [Available from: https://www.drugbank.ca/drugs/DB12129.

67. Database d. PX-12 2020,March 1 [Available from: https://www.drugbank.ca/drugs/DB05448.

68. CaymanChem. TDZD-8 2020,April 12 [Available from: https://www.caymanchem.com/product/16287/tdzd8.

69. Medscape. Parasite Drug Shows Early Promise Against COVID-19 in Vitro 2020,April 8 [Available from: https://www.medscape.com/viewarticle/928345.

70. Database D. Bepotastine. 2020,April 11.

71. Medical Press. Many drugs already approved by FDA may have promise against COVID-19 2020,March 24 [Available from: https://medicalxpress.com/news/2020-03-drugs-fda-covid-.html.

72. Xu K, Cai H, Shen Y, Ni Q, Chen Y, Hu S, et al. Management of corona virus disease-19 (COVID-19): the Zhejiang experience. Zhejiang da xue xue bao Yi xue ban = Journal of Zhejiang University Medical sciences. 2020;49(1):0.

73. Du Y, Tu L, Zhu P, Mu M, Wang R, Yang P, et al. Clinical Features of 85 Fatal Cases of COVID-19 from Wuhan: A Retrospective Observational Study. American Journal of Respiratory and Critical Care Medicine.0(ja):null.

74. Zhang H, Huang Y, Xie C. The Treatment and Outcome of a Lung Cancer Patient Infected with SARSCoV-2. Journal of thoracic oncology : official publication of the International Association for the Study of Lung Cancer. 2020.

75. Chen L, Xiong J, Bao L, Shi Y. Convalescent plasma as a potential therapy for COVID-19. The Lancet Infectious diseases. 2020.

76. Zhang B, Liu S, Tan T, Huang W, Dong Y, Chen L, et al. Treatment with convalescent plasma for critically ill patients with SARS-CoV-2 infection. Chest. 2020.

77. Keith P, Day M, Perkins L, Moyer L, Hewitt K, Wells A. A novel treatment approach to the novel coronavirus: an argument for the use of therapeutic plasma exchange for fulminant COVID-19. Critical Care. 2020;24(1):128. 